\title{
Energy Efficiency Maximization for Energy Harvesting Bidirectional Cooperative Sensor Networks with AF Mode
}

\author{
Siyang $\mathrm{Xu}^{1,2}$, Xin Song ${ }^{1,2^{*}}$, Lin $\mathrm{Xia}^{1,2}$ and Zhigang $\mathrm{Xie}^{1,2}$ \\ ${ }^{1}$ School of Computer Science and Engineering, Northeastern University, \\ Shenyang 110819, China \\ ${ }^{2}$ Engineering Optimization and Smart Antenna Institute, \\ Northeastern University at Qinhuangdao, Qinhuangdao 066004, China \\ [e-mail: 1810584@stu.neu.edu.cn, sxin78916@neuq.edu.cn,804816798@qq.com, Zhigangxie@foxmail.com] \\ *Corresponding author: Xin Song
}

Received November 13, 2019; revised March 27, 2020; accepted April 6, 2020; published June 30, 2020

\begin{abstract}
This paper investigates the energy efficiency of energy harvesting (EH) bidirectional cooperative sensor networks, in which the considered system model enables the uplink information transmission from the sensor (SN) to access point (AP) and the energy supply for the amplify-and-forward (AF) relay and SN using power-splitting (PS) or time-switching (TS) protocol. Considering the minimum $\mathrm{EH}$ activation constraint and quality of service (QoS) requirement, energy efficiency is maximized by jointly optimizing the resource division ratio and transmission power. To cope with the non-convexity of the optimizations, we propose the low complexity iterative algorithm based on fractional programming and alternative search method (FAS). The key idea of the proposed algorithm first transforms the objective function into the parameterized polynomial subtractive form. Then we decompose the optimization into two convex sub-problems, which can be solved by conventional convex programming. Simulation results validate that the proposed schemes have better output performance and the iterative algorithm has a fast convergence rate.
\end{abstract}

Keywords: Cooperative sensor network, energy efficiency, power splitting protocol, time switching protocol, amplify-and-forward relay

This work was supported in part by the National Nature Science Foundation of China under Grant 61473066 and Grant 61601109, and in part by the Fundamental Research Funds for the Central Universities under Grant No. N152305001, and in part by the Natural Science Foundation of Hebei Province under Grant F2017501039. 


\section{Introduction}

$\mathbf{D}_{\text {ue to the limited transmission range of wireless sensor networks, cooperative technologies }}$ can be used to expend coverage and achieve higher spatial diversity, in which the relay node is played by an idle sensor [1, 2]. However, the sensors are generally placed in the human body or special environment. In actual operation, it is inconvenient and expensive to recharge or replace the batteries [3]. Furthermore, the cooperative sensors may be reluctant to consume their energy to assist the transmission, which results in the power-constrained problems. For this difficulty, EH is a new green technology to provide continuous power supply for the wireless devices by harvesting energy from ambient environment. Besides traditional EH sources such as solar, wind and heat, a new solution is to exploit the energy carried by radio frequency (RF) signals [4]. Subsequently, two major EH relay protocols named as "time switching (TS) protocol" and "power splitting (PS) protocol" are proposed for cooperative networks [5]. For the TS relay protocol, the total transmission process can be divided into three phases, which are used for $\mathrm{EH}$, information receiving and information relaying respectively. For the PS relay protocol, the relay nodes split the received signal into two streams with different power to achieve energy supply and information decoding [6]. In conventional EH one-way relay systems, the throughput maximization is investigated with amplify-and-forward (AF) and decode-and-forward (DF) relay mode [7-9]. Furthermore, EH technologies are applied to the internet of things and cognitive networks to solve the power-constrained problems of wireless devices $[10,11]$. In [12, 13], the time resource assignment for the backscatter-aided RF powered cognitive radio networks is investigated. On the other hand, due to the broadcast nature of RF energy transmission, system security will not be guaranteed. Therefore, an artificial-noise-aided beamforming design is proposed for a downlink multi-input single-output (MISO) EH network and a two-tier heterogeneous EH cellular network [14, 15]. In [16], the minimum transmission power optimization guaranteeing the secrecy rate constraint and the transmit power constraint is solved by using the S-procedure and semidefinite relaxation techniques, in which the relay can utilize the PS or TS relay protocol to harvest energy.

Comparing with one-way relay systems, two-way relaying has been widely used in various EH networks owing to its high spectrum efficiency. Time division broadcast (TDBC) and multiple access broadcast (MABC) are two major two-way relay protocols, which can realize that two terminals exchange information through an EH intermediate relay [17-26]. For TDBC $\mathrm{EH}$ protocol, the total transmission process is divided into three unequal length slots, and the relay node can harvest energy from two sources in the first two time phases [17, 18]. In [19-21], energy efficiency and outage performance are optimized with DF relay mode. For the MABC EH protocol, the relay node can harvest energy from two sources in the same phases [22-26]. Under PS protocol, the optimal power allocation and relay selection schemes based on max-min rate of two links are designed with perfect and imperfect channel state information (CSI) [22, 23]. And the joint power allocation and relay selection scheme based on the TS protocol is proposed in [24]. Taking the sum rate as the performance metric, the power allocation schemes are also investigated in two-way EH networks [25, 26].

In some typical sensor networks, remote sensors need to transmit real-time information to the AP through the relay node, in which both sensors and relay nodes are energy-constrained. Inspired by the traditional two-way relay networks, the EH bidirectional cooperative system networks are proposed to solve this problem, in which both the SN and relay can be charged 
via the wireless powered transfer [27-30]. In [27, 28], the instantaneous rate maximization problems with respect to the resource division ratio are studied with the situations of one and multiple DF relay nodes. As the extension of [27, 28], the energy efficiency maximization optimization is investigated in [29]. However, the joint optimization problem in [29] is not solved due to the non-convexity of the objective function. Besides, the basic QoS constraints and $\mathrm{EH}$ activation constraints in energy efficiency optimization problems are also ignored. Using the AF relay node, the optimal closed-form resource division ratio can be obtained to maximize the instantaneous rate under the special case $P_{A} \rightarrow \infty$ [30].

With the development of the concepts of environmental protection and sustainable development, energy efficiency has been an important metric to evaluate the network performance of cellular mobile communication systems [31, 32]. And a joint optimization problem of computation and communication power is formulated for multi-user massive MIMO systems with partially-connected structures of RF transmission systems [33]. However, most energy efficiency maximization problems are non-convex. To cope with these problems, the iterative algorithms are proposed to transform the original non-convex problem into the convex problems step by step [34]. Using fractional programming, the fractional form of the objective function can be transferred to a parameterized polynomial subtractive form, which can be solved by Dinkelbach's method [35]. The non-convex constraints can be integrated into the objective function by Lagrange dual method [36] or exact penalty method [37]. In addition, the alternative search method can decompose the optimization into sub-problems that are easy to solve [38]. Hence, the above optimization algorithm has great inspiration for solving non-convex optimizations of this paper.

The main contributions of this paper are summarized as follows:

1. In this paper, we investigate the energy efficiency of EH bidirectional cooperative sensor networks with AF mode, in which the relay node can assist uplink information transmission from the SN to AP and the downlink energy transmission from the AP to SN. The considered system model can solve the power-constrained of the relay and SN using TS or PS protocol. Furthermore, we also consider the imperfect self-interference cancellation (SIC) with the PS relay protocol, which is more in line with the actual application scenario. To maximize the energy efficiency, the joint optimizations of the transmission power and resource division ratio are formulated subject to the $\mathrm{EH}$ activation and QoS requirement.

2. For the formulated non-convex optimizations, we propose the low complexity FAS iterative algorithm based on fractional programming and alternative search method to obtain the global optimal transmission power and resource division ratio. The key idea of the proposed algorithm first converts the objective function into the parameterized polynomial subtractive form by fractional programming. However, the converted optimizations are still non-convex. Hence, we decompose the optimizations into two sub-problems, which can be proved to be convex. The local solutions can be obtained by conventional convex programming and the global solutions can be obtained by the alternative search method.

3. Simulation results illustrate the convergence and low complexity of the proposed FAS algorithm. Furthermore, we compare the proposed schemes with other conventional schemes, which show that the proposed schemes can provide more performance gain over conventional schemes.

The rest of this paper is organized as follows. The proposed EH bidirectional cooperative sensor networks are described in Section 2. Considering the EH activation constraint and the minimum target rate requirement, the energy efficiency maximization problems are formulated. And a distributed iterative algorithm with low complexity is proposed to solve the 
non-convex optimization in Section 3. Simulation results verify the effectiveness of the proposed resource allocation strategies in Section 4. Section 5 concludes this paper.

\section{System Model}

The considered EH bidirectional cooperative sensor network is depicted in Fig. 1, in which the SN sends the information to the AP with the help of an AF relay. All nodes are equipped with a single antenna for half-duplex operation. The relay node is acted by the idle sensor, which serves dual roles to achieve both energy and information relaying. The relay node and SN are power-constrained and solely powered by $\mathrm{EH}$, while the AP can be powered by on-grid power. All the harvested energy of SN and relay is consumed for information transmission. To maintain the switching between wireless power and information transmission, the storage unit is equipped at both relay and $\mathrm{SN}$, which is a rechargeable battery. Using the existing energy of the SN battery can initialize the information transmission before EH [29]. Due to the long-distance and deep fading, the direct link between AP and SN is ignored. Moreover, it is assumed that the quasi-static channel model with perfect CSI can be obtained by the transmitters. In practice, the CSI can be acquired by the pilot-assisted reverse-link channel training [30].

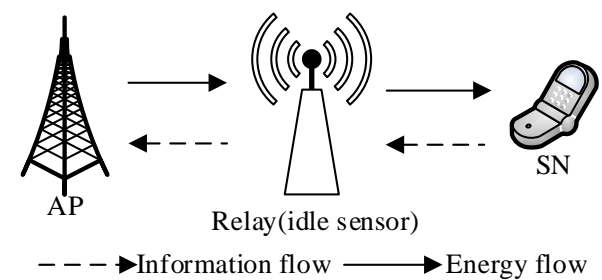

Fig. 1. The system model of bidirectional cooperative sensor networks.

\subsection{Power Splitting Protocol}

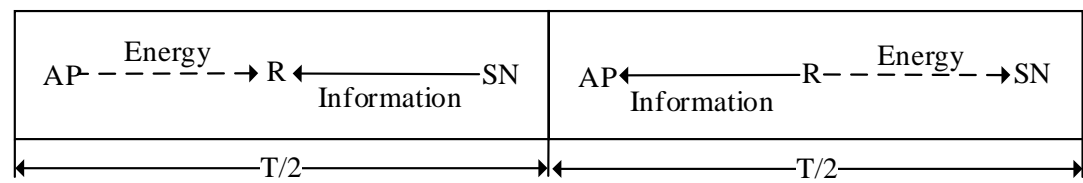

Fig. 2. Bidirectional cooperative sensor networks with PS protocol.

For PS protocol, the entire transmission duration of the bidirectional relay system is completed within two equal phases $T / 2$. As illustrated in Fig. 2, the relay receives the energy signal $x_{a}$ and information signal $x_{s}$ transmitted from the AP and SN during the first phase. Therefore, the received signal of the relay can be given by

$$
y_{R}=\sqrt{P_{A}} h x_{a}+\sqrt{P_{S}} g x_{s}+n_{R} .
$$

where $P_{S}$ and $P_{A}$ denote the transmission powers of the SN and AP; the channel gains from AP and SN to the relay node are $h$ and $g$, which are assumed to be reciprocal; $n_{R}$ is the additive white Gaussian noise at the relay node. 
Since we adopt the PS protocol, the relay node assigns $\rho$ portion to recharge its battery, and the other $1-\rho$ portion is used for energy and information relaying, where $\rho \in(0,1)$ is the PS factor. In practice, the SN harvests the energy transmitted from AP via the relay node. The energy signal experiences two times channel attenuations and a series of other losses, which results in $P_{S}<<P_{A}$ [27-30]. This phenomenon can be explained by equation (10). Thus, we ignore the harvested energy from SN and the noise. During the first phase, the harvested energy of the relay node can be given as

$$
E_{R}=\xi \rho \mathrm{E}\left\{\left|y_{R}\right|^{2}\right\}(T / 2) \approx \xi \rho P_{A}|h|^{2}(T / 2),
$$

where $\mathrm{E}\{\cdot\}$ represents the expectation operation; $\xi \in(0,1)$ is the energy conversion efficiency. The transmission power of the relay node can be calculated as

$$
P_{R}=\xi \rho P_{A}|h|^{2}
$$

Then, the remaining $1-\rho$ portion signal can be given by

$$
y_{R}^{\prime}=\sqrt{1-\rho}\left(\sqrt{P_{A}} h x_{a}+\sqrt{P_{S}} g x_{S}+n_{R}\right) .
$$

In the second phase of the time duration $T / 2$, the relay node broadcasts energy and information to the SN and AP. To use more energy for the information signal relaying, the relay cancels the energy signal $x_{a}$ from $y_{R}^{\prime}$ by using SIC. In the case of imperfect cancelation, the post-cancellation signal at the relay can be expressed as

$$
\hat{y}_{R}=\sqrt{1-\rho}\left(\sqrt{\zeta P_{A}} h x_{a}+\sqrt{P_{S}} g x_{S}+n_{R}\right) \text {, }
$$

where $\zeta \in(0,1]$ is defined as the cancelation coefficient to characterize the level of imperfect cancelation. Therefore, the signal transmitted by the relay node can be given by

$$
x_{R}=\sqrt{\beta}\left(\sqrt{1-\rho}\left(\sqrt{P_{S}} g x_{S}+\sqrt{\zeta P_{A}} h x_{a}+n_{R}\right)+n_{R}^{\prime}\right),
$$

where $\beta$ denotes the normalized amplify factor; $n_{R}^{\prime} \sim C N\left(0, \sigma_{R}^{2}\right)$ is the additional processing noise. Since $n_{R}^{\prime}$ dominates the antenna noise $n_{R}$, we ignore $n_{R}$ for simplicity. The signal transmitted by the relay node can be re-represented as

$$
x_{R}=\sqrt{\beta}\left(\sqrt{1-\rho} \sqrt{P_{S}} g x_{S}+\sqrt{\zeta(1-\rho)} \sqrt{P_{A}} h x_{a}+n_{R}^{\prime}\right) .
$$

With the given expression in (5), the normalized amplify factor can be given by 


$$
\beta=\frac{\xi \rho P_{A}|h|^{2}}{(1-\rho) P_{S}|g|^{2}+(1-\rho) \zeta P_{A}|h|^{2}+\sigma_{R}^{2}} .
$$

Subsequently, $x_{R}$ is amplified and forwarded to the AP. Thus, the received information signal of AP can be expressed as

$$
y_{A}=h \sqrt{\beta}\left(\sqrt{1-\rho} \sqrt{P_{S}} g x_{S}+\sqrt{\zeta(1-\rho)} \sqrt{P_{A}} h x_{a}+n_{R}^{\prime}\right)+n_{A},
$$

where $n_{A} \sim C N\left(0, \sigma_{A}^{2}\right)$ denotes the additive noise of AP.

On the other hand, the received energy signal at SN can be given by $y_{S}=g x_{R}$. Ignoring the additive noise at $\mathrm{SN}$, the transmission power of SN can be calculated as

$$
P_{S}=\frac{\xi|g|^{2} \mathrm{E}\left\{\left|x_{R}\right|^{2}\right\}}{T / 2}=\xi^{2} P_{A} \rho|h|^{2}|g|^{2} .
$$

Combining the equations (8), (9) and (10), the received signal-to-noise ratio (SNR) at AP is formulated as

$$
\begin{aligned}
& \gamma_{P S}\left(P_{A}, \rho\right)=\frac{\beta(1-\rho) P_{S}|h|^{2}|g|^{2}}{\beta|h|^{2} \sigma_{R}^{2}+\beta \zeta(1-\rho) P_{A}|h|^{4}+\sigma_{A}^{2}} \\
& =\frac{\xi^{3} P_{A}^{2} \rho^{2}(1-\rho)|h|^{6}|g|^{4}}{\xi \rho P_{A}|h|^{4} \sigma_{R}^{2}+\xi \zeta \rho(1-\rho) P_{A}{ }^{2}|h|^{6}+\left((1-\rho) \xi^{2} P_{A} \rho|h|^{2}|g|^{4}+(1-\rho) \zeta P_{A}|h|^{2}+\sigma_{R}^{2}\right) \sigma_{A}^{2}}
\end{aligned}
$$

As a result, the instantaneous information rate at AP with respect to $\rho$ and $P_{A}$ is

$$
R_{P S}\left(P_{A}, \rho\right)=\frac{1}{2} \log _{2}\left(1+\gamma_{P S}\left(P_{A}, \rho\right)\right) .
$$

The total transmission process of the PS relay protocol is divided into two equal durations, while the AP only transmits power $P_{A}$ during the first phase. Besides, part of the energy $(1-\rho)(1-\zeta) P_{A}$ is canceled in the second phase. Note that the total power consumption of the relay node and the SN is supplied by the AP. Thus, the total power consumption of the considered network with PS protocol can be given by

$$
P_{\text {total }}^{P S}=\frac{1}{2} \kappa P_{A}+P_{c}-\frac{1}{2}(1-\zeta)(1-\rho) P_{A}=\frac{1}{2}(\kappa-(1-\rho)(1-\zeta)) P_{A}+P_{c},
$$

where $\kappa$ is the inverse of power amplification efficiency; $P_{c}$ is the total circuit power consumption by digital to analog converter and frequency synthesizer. 


\subsection{Time Switching Protocol}

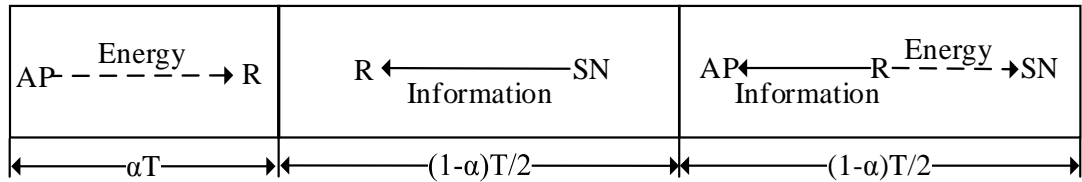

Fig. 3. Bidirectional cooperative sensor networks with TS protocol.

The TS protocol requires three phases to complete the entire transmission, which is shown in Fig. 3. During the first phase $\alpha T$, the energy signal with power $P_{A}$ is transmitted from the AP to the relay node, where $\alpha \in(0,1)$ denotes the TS factor. Thus, the amount of harvested energy at the relay node can be given by

$$
E_{R} \approx \xi P_{A}|h|^{2} \alpha T
$$

In the second phase of duration $(1-\alpha) T / 2, \mathrm{SN}$ transmits its information $x_{s}$ to the relay. The received signal at the relay node can be expressed as

$$
y_{R}=\sqrt{P_{S}} g x_{S}+n_{R} .
$$

Using all the harvested energy $E_{R}$, the relay sends the amplified signal $\sqrt{\beta} y_{R}$ to the AP and the $\mathrm{SN}$ in the remaining phase, where the normalized amplify factor can be given by

$$
\beta=\frac{2 \xi \alpha P_{A}|h|^{2}}{(1-\alpha)\left(P_{S}|g|^{2}+\sigma_{R}^{2}\right)} .
$$

The received signal at the AP is

$$
y_{A}=h \sqrt{\beta}\left(\sqrt{P_{S}} g x_{S}+n_{R}\right)+n_{A} .
$$

Hence, the harvested energy at the SN can be calculated as $E_{S}=\xi|g|^{2} \beta \mathrm{E}\left[\left|y_{R}\right|^{2}\right](1-\alpha) T / 2$ $=\xi^{2} P_{A}|h|^{2}|g|^{2} \alpha T$. And the transmission power of the SN can be given by

$$
P_{S}=\frac{E_{S}}{(1-\alpha) / 2}=\frac{2 \xi^{2} P_{A}|h|^{2}|g|^{2} \alpha}{1-\alpha}
$$

Combining the equations (16), (17) and (18), the received SNR at AP can be calculated as 


$$
\begin{aligned}
\gamma_{T S}\left(P_{A}, \alpha\right)=\frac{\beta P_{S}|h|^{2}|g|^{2}}{\beta|h|^{2} \sigma_{R}^{2}+\sigma_{A}^{2}}= & \frac{4 \xi^{3} \alpha^{2} P_{A}^{2}|h|^{6}|g|^{4}}{2 \xi \alpha(1-\alpha) P_{A}|h|^{4} \sigma_{R}^{2}} . \\
& +\left(2(1-\alpha) \xi^{2} P_{A}|h|^{2}|g|^{4} \alpha^{2}+(1-\alpha)^{2} \sigma_{R}^{2}\right) \sigma_{A}^{2}
\end{aligned}
$$

As a result, the instantaneous information rate about $\alpha$ and $P_{A}$ is

$$
R_{T S}\left(P_{A}, \alpha\right)=\frac{1-\alpha}{2} \log _{2}\left(1+\gamma_{T S}\left(P_{A}, \alpha\right)\right)
$$

The total transmission process of the TS relay protocol is divided into three phases, while the AP only transmits the power in the first phase duration $\alpha T$. And all the energy of the relay and SN are originated from AP. Therefore, the total power consumption can be given by

$$
P_{\text {total }}^{T S}=\alpha \kappa P_{A}+P_{c}
$$

\section{Formulation and Solution of Energy Efficiency Optimization}

In this section, the energy efficiency maximization optimizations subject to EH activation constraint and QoS requirement are formulated under the PS and TS protocol. Due to the transmission power and resource division factor are coupled to each other, we can not obtain the global optimal solutions directly. To cope with the non-convexity of the optimizations, the FAS algorithms are proposed. By using the fractional programming, the original objective function is converted into parameterized polynomial subtractive form. Then the transferred optimizations can be decomposed into two sub-problems. After proving that the optimization is a convex problem with the given resource division ratio or transmission power, the global solutions can be obtained by the alternative search method.

\subsection{Power Splitting Protocol}

\subsubsection{Problem Formulation}

The definition of energy efficiency is the ratio of the information rate and the total power consumption, which is given by

$$
\eta_{e e}=\frac{R}{P_{\text {total }}}[\text { bits / Joule }]
$$

In the case of high SNR, the equation (11) can be approximated as

$$
\gamma_{P S}\left(P_{A}, \rho\right) \approx \frac{\xi^{3} P_{A} \rho^{2}(1-\rho)|h|^{6}|g|^{4}}{\xi \rho|h|^{4} \sigma_{R}^{2}+\xi \zeta \rho(1-\rho) P_{A}|h|^{6}+\left((1-\rho) \xi^{2} \rho|h|^{2}|g|^{4}+(1-\rho) \zeta|h|^{2}\right) \sigma_{A}^{2}} .
$$

Based on (22), the energy efficiency of PS relay protocol $\eta_{e e}^{P S}$ is formulated as 


$$
\eta_{e e}^{P S}\left(P_{A}, \rho\right)=\frac{R_{P S}\left(P_{A}, \rho\right)}{P_{\text {total }}^{P S}\left(P_{A}, \rho\right)}=\frac{\frac{1}{2} \log _{2}\left(1+\gamma_{P S}\left(P_{A}, \rho\right)\right)}{\frac{1}{2}(\kappa-(1-\rho)(1-\zeta)) P_{A}+P_{c}} .
$$

Considering the QoS requirement and EH activation constraint, the optimization of energy efficiency maximization can be given by

$$
\begin{aligned}
& \text { P1: } \max _{\left\{P_{A}, \rho\right\}} \eta_{e e}^{P S}\left(P_{A}, \rho\right) \\
& \text { s.t. } C_{1} R_{P S}>R_{t h} \\
& C_{2} \quad 0<\rho \leq 1 \\
& C_{3} \quad 0<P_{A} \leq P_{\text {max }} \\
& C_{4} P_{A}|h|^{2}>\theta \\
& C_{5} \xi \rho P_{A}|h|^{2}|g|^{2}>\theta
\end{aligned}
$$

where $R_{t h}$ is the minimum rate requirement; $\theta$ represents the activation sensitivity of EH receiver; $P_{\max }$ is the maximum power of the AP. Besides, $P_{A}|h|^{2}$ and $\xi \rho P_{A}|h|^{2}|g|^{2}$ are the power arrived at the relay and SN. Obviously, both the objective function and constraint $C_{1}$ are non-convex due to $\rho$ and $P_{A}$ are coupled to each other, which results in the non-convexity of the optimization problem.

\subsubsection{The Proposed Iterative Algorithm for PS Protocol}

For the proposed FAS algorithm, we first transform the objective function into parametric programming, which can be solved by Dinkelbach's method. Let $\varphi^{*}$ denotes the maximum energy efficiency of the considered system. Then we have that

$$
\varphi^{*}=\frac{R_{P S}\left(P_{A}^{*}, \rho^{*}\right)}{P_{\text {total }}^{P S}\left(P_{A}^{*}, \rho^{*}\right)}=\max _{P_{A}, \rho} \frac{R_{P S}\left(P_{A}, \rho\right)}{P_{\text {total }}^{P S}\left(P_{A}, \rho\right)},
$$

where $P_{A}{ }^{*}$ and $\rho^{*}$ are the optimal transmission power and PS factor. Since $\varphi^{*}, P_{A}{ }^{*}$ and $\rho^{*}$ are the optimal solutions, we have

$$
\varphi^{*}=\frac{R_{P S}\left(P_{A}^{*}, \rho^{*}\right)}{P_{\text {total }}^{P S}\left(P_{A}^{*}, \rho^{*}\right)} \geq \frac{R_{P S}\left(P_{A}, \rho\right)}{P_{\text {total }}^{P S}\left(P_{A}, \rho\right)} .
$$

After the mathematical operation, (27) can be rewritten as

$$
R_{P S}\left(P_{A}, \rho\right)-\varphi^{*} P_{\text {total }}^{P S}\left(P_{A}, \rho\right) \leq 0,
$$




$$
R_{P S}\left(P_{A}^{*}, \rho^{*}\right)-\varphi^{*} P_{\text {total }}^{P S}\left(P_{A}^{*}, \rho^{*}\right)=0
$$

Therefore, the upper boundary of $R_{P S}\left(P_{A}, \rho\right)-\varphi^{*} P_{\text {total }}^{P S}\left(P_{A}, \rho\right)$ is equal to 0 . In other words, the maximum energy efficiency $\varphi^{*}$ can be achieved only when the following equation is satisfied

$$
\max _{\left\{P_{A}, \rho\right\}} R_{P S}\left(P_{A}, \rho\right)-\varphi^{*} P_{\text {total }}^{P S}\left(P_{A}, \rho\right)=R_{P S}\left(P_{A}^{*}, \rho^{*}\right)-\varphi^{*} P_{\text {total }}^{P S}\left(P_{A}^{*}, \rho^{*}\right)=0 .
$$

where

$$
\max _{\left\{P_{A}, \rho\right\}} R_{P S}\left(P_{A}, \rho\right)-\varphi P_{\text {total }}^{P S}\left(P_{A}, \rho\right)
$$

is defined as the parametric program with parameter $\varphi$.

Remark 1: In fact, using Dinkelbach's method can generate a strictly increasing sequence $\varphi(i)$ that superlinearly converges to $\varphi^{*}$ with an initial value $\varphi(0)<\varphi^{*}$. The convergence of fractional programming has been proved in [39, 40]. Hence, after finite iterations, the iterative process will terminate at $R_{P S}\left(P_{A}(i), \rho(i)\right)-\varphi(i) P_{\text {total }}^{P S}\left(P_{A}(i), \rho(i)\right)<\omega$ with the convergence tolerance $\omega>0$. Based on the above analysis, the algorithm of fractional programming is given in Algorithm 1 , where $I$ is the maximum number of iterations.

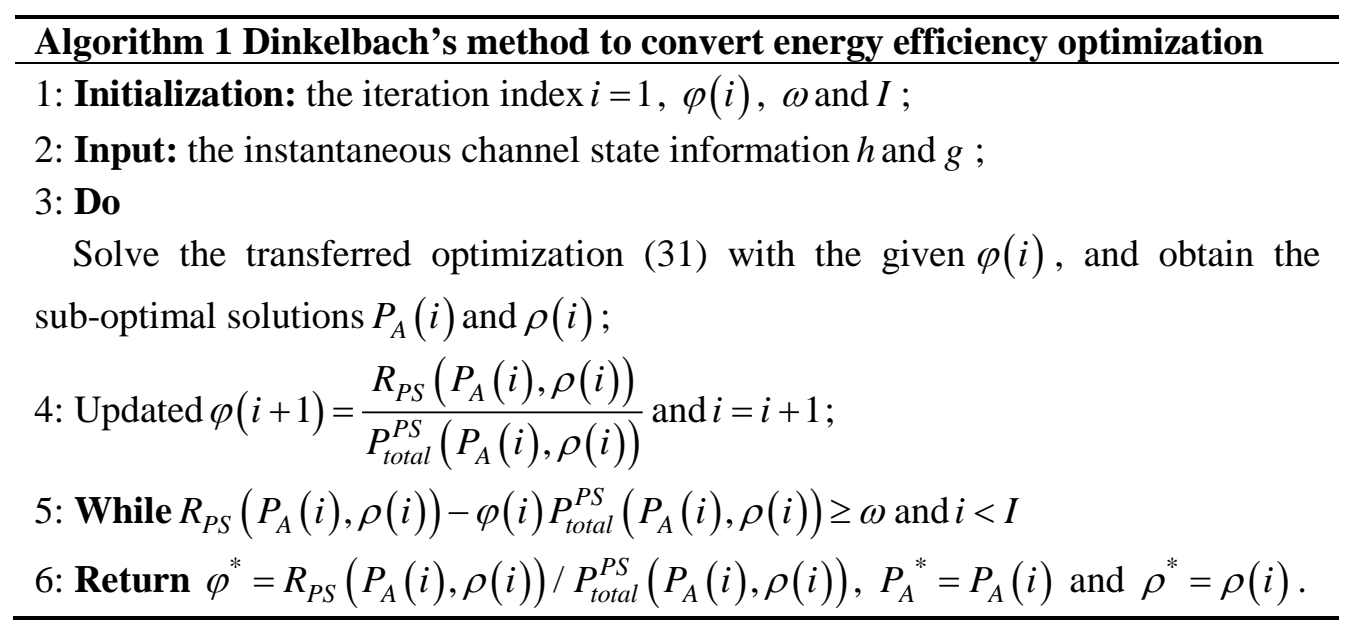

After applying the fractional programming, the optimization problem $P 1$ can be re-expressed as

$$
\begin{aligned}
P 1 a: & \max _{\left\{P_{A}, \rho\right\}} R_{P S}\left(P_{A}, \rho\right)-\varphi P_{\text {total }}^{P S}\left(P_{A}, \rho\right) \\
\text { s.t. } C_{1} \sim C_{5} & .
\end{aligned}
$$

However, the optimization problem is still non-convex. We still cannot obtain the final solution by mathematical calculations directly. To solve this problem, we decompose $P 1 a$ into two sub-problems with fixed $P_{A}$ or $\rho$. 
Proposition 1. With a given $\rho, P 1 a$ is a convex problem with respect to $P_{A}$. When $P_{A}$ is given, $P 1 a$ is also a convex problem with respect to $\rho$.

Proof: See Appendix A.

After proving the convexity of the decomposed two sub-problems, the local solutions can be obtained by convex programming. Then, the alternative search method can be used to obtain the global optimal solutions iteratively. The key idea of the alternative convex programming is that only one local solution of $\rho$ or $P_{A}$ can be obtained in each iteration while the other is fixed, which is shown in Algorithm 2. The convergence tolerance and the maximum number of iterations are denoted by $\varpi$ and $K$.

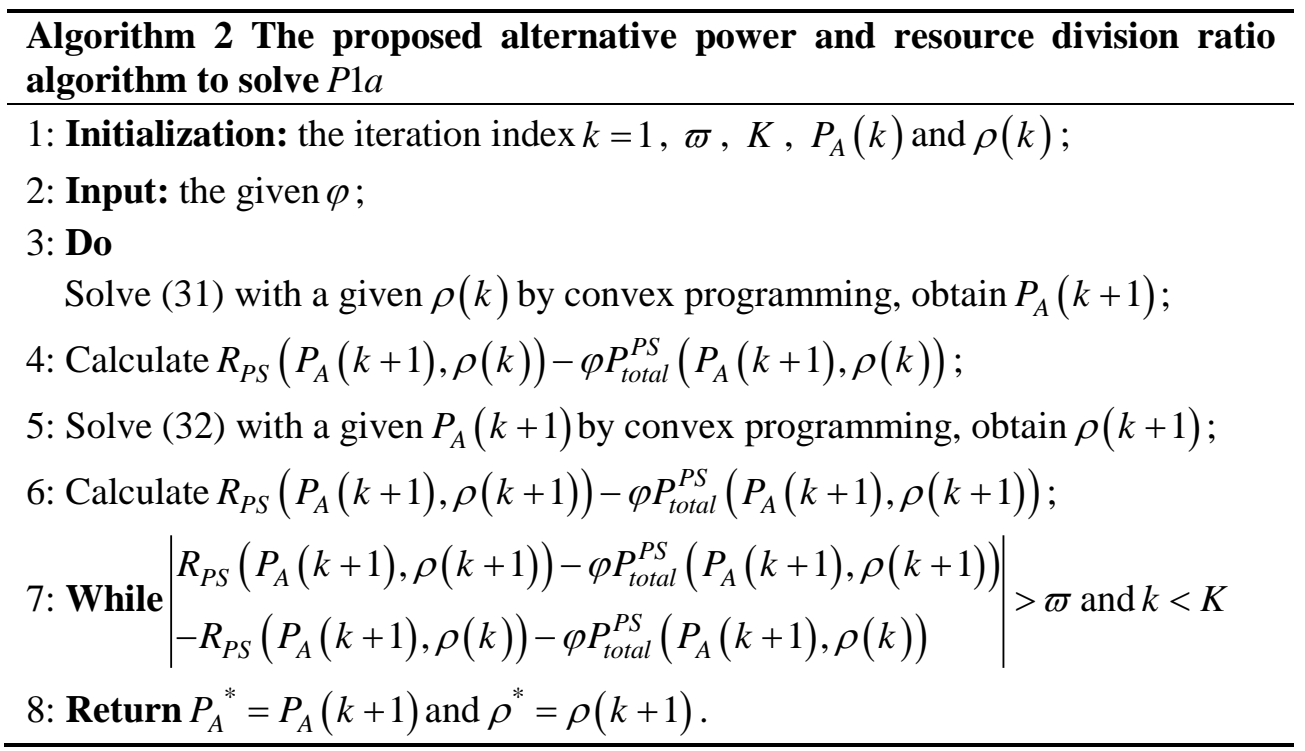

Proposition 2. The local solutions can be obtained by convex programming. Then, the generated sequence $R_{P S}\left(P_{A}(k+1), \rho(k+1)\right)-\varphi P_{\text {total }}^{P S}\left(P_{A}(k+1), \rho(k+1)\right)$ by the alternative search method converges monotonically.

Proof: See Appendix B.

\subsection{Time Switching Protocol}

\subsubsection{Problem Formulation}

In the case of high SNR, the energy efficiency $\eta_{e e}^{T S}$ can be formulated as

$$
\eta_{e e}^{T S}\left(P_{A}, \alpha\right)=\frac{R_{T S}\left(P_{A}, \alpha\right)}{P_{\text {total }}^{T S}\left(P_{A}, \alpha\right)}=\frac{\frac{1-\alpha}{2} \log _{2}\left(1+\frac{2 \xi P_{A}|h|^{4}|g|^{4} \alpha}{(1-\alpha)\left(|h|^{2} \sigma_{R}^{2}+\xi|g|^{4} \sigma_{A}^{2}\right)}\right)}{\alpha \kappa P_{A}+P_{c}} .
$$

Thus, the energy efficiency maximization problem with consideration of the QoS requirement and EH activation constraint can be expressed as 


$$
\begin{array}{cl}
\text { P2: } \max _{\left\{P_{A}, \alpha\right\}} \eta_{e e}^{T S}\left(P_{A}, \alpha\right) \\
\text { s.t. } C_{1} \quad R_{T S}>R_{t h} \\
C_{2} \quad 0<\alpha \leq 1 \\
C_{3} \quad 0<P_{A} \leq P_{\max } \\
C_{4} \quad P_{A}|h|^{2}>\theta \\
C_{5} \quad \frac{2 \xi \alpha P_{A}|h|^{2}|g|^{2}}{(1-\alpha)}>\theta
\end{array}
$$

\subsubsection{The Proposed Iterative Algorithm for TS Protocol}

To solve the non-convexity of $P 2$, the fractional programming can be used to convert (33) into a parameterized subtractive form, which can be expressed as

$$
\begin{gathered}
P 2 a: \max _{\left\{P_{A}, \alpha\right\}} R_{T S}\left(P_{A}, \alpha\right)-\delta P_{\text {total }}^{T S}\left(P_{A}, \alpha\right), \\
\text { s.t. } C_{1} \sim C_{5}
\end{gathered}
$$

where $\delta$ is the parameter of the fractional programming. It is obvious that $P 2 a$ is still a non-convex problem. Similar to the PS scheme, we decompose the optimization (36) into two sub-problems with respect to $P_{A}$ and $\alpha$ respectively.

Proposition 2. With a given $\alpha, P 2 a$ is a convex problem with respect to $P_{A}$. When $P_{A}$ is given, $P 2 a$ is also a convex problem with respect to $\alpha$.

Proof: See Appendix C.

Then the alternative convex programming can be used to obtain global solutions. Changing the optimization parameters and objective function, the outer loop can be solved by Algorithm 1, and the inner loop can be solved by Algorithm 2. The convergence of the proposed algorithm with TS relay protocol is similar to the PS relay protocol. Thus, the proof is omitted here.

\subsection{Computational Complexity Analysis}

In this section, the computational complexity of the proposed algorithm is analyzed. The proposed algorithm is a nested structure with fractional programming, alternative search method and convex programming. The global solutions of transmission power and resource division ratio can be obtained by the proposed FAS algorithm with low computational complexity. Except for the convex programming part, the total number of iterations can be given by $\min \left\{\omega_{i} \varpi_{k}, I K\right\}$, where $\omega_{i}$ and $\varpi_{k}$ denote the iterative numbers corresponding to the fractional programming and alternative search method when the stop conditions $\omega$ and $\varpi$ are reached. The final convex sub-problems can be solved by the fast gradient method [41]. Hence, the computational complexity of the fast gradient method with respect to transmission power and resource division ratio can be given by $\psi_{1}=O(1) \min \left\{\sqrt{\frac{\varsigma_{1}}{\tau_{1}}} \ln \left(\frac{1}{v_{1}}\right), \sqrt{\frac{\varsigma_{1}}{v_{1}}}\right\}$ and $\psi_{2}=O(1) \min \left\{\sqrt{\frac{\varsigma_{2}}{\tau_{2}}} \ln \left(\frac{1}{v_{2}}\right), \sqrt{\frac{\varsigma_{2}}{v_{2}}}\right\}$, where $\varsigma_{1}$ and $\varsigma_{2}$ are the Lipschitz constants; $\tau_{1}$ and $\tau_{2}$ are 
the convexity parameters; $v_{1}$ and $v_{2}$ are the convergence tolerances, referring to [41]. Therefore, the overall computational complexity of the proposed algorithm can be given by $\left\{\min \left\{\omega_{i} \varpi_{k}, I K\right\}\right\}\left(\psi_{1}+\psi_{2}\right)$.

\section{Simulation Results}

In this section, we first prove the convergence and low complexity of the proposed iterative algorithm comparing with the exhaustive search method. Subsequently, we compare the proposed schemes with traditional schemes under different parameters to evaluate energy efficiency performance. For the proposed schemes, we assume that the distance between AP and SN are fixed at $d_{A S}=3 \mathrm{~m}$, and the relay can move within the line of AP and SN, where $d_{A S}$, $d_{A R}$ and $d_{R S}$ denote the distance between AP to SN, AP to relay and relay to SN respectively. The channel gain $|h|^{2}$ and $|g|^{2}$ are set to $d_{A R}{ }^{-\lambda}$ and $d_{R S}{ }^{-\lambda}$ [27], where $\lambda$ is the path-loss exponent. And the simulation parameters are presented in Table 1.

Table 1. Simulation parameters

\begin{tabular}{cc}
\hline Parameter & Value \\
\hline Path-loss exponent $\lambda$ & 3 \\
Cancellation coefficient $\zeta$ & 0.001 \\
Energy conversion efficiency $\xi$ & 0.3 \\
Noise power density $\sigma_{R}^{2}, \sigma_{A}^{2}$ & $-10 \mathrm{dBm} / \mathrm{Hz}$ \\
Total static power consumption $P_{c}$ & $25 \mathrm{~mW}$ \\
The inverse of power amplification efficiency $\kappa$ & 1 \\
Maximum transmission power $P_{\max }$ & $30 \mathrm{dBm}$ \\
Activation threshold of EH circuit $\theta$ & $-10 \mathrm{dBm}$ \\
The minimum rate requirement $R_{t h}$ & $1 \mathrm{bps} / \mathrm{Hz}$ \\
Convergence tolerance of iterative algorithms $\omega, \varpi$ & $10^{-5}$ \\
\hline
\end{tabular}

For evaluating the output performance and the convergence behavior, the energy efficiency of the proposed PS and TS schemes are illustrated in Fig. 4 and Fig. 5 by contrast with the exhaustive search method. The distance between AP to relay $d_{A S}$ is set as $1.5 \mathrm{~m}$. The step-size of the exhaustive search accuracy is set as $10^{-5}$, which is not strictly accurate but results in a great amount of calculation. Simultaneously, the global solutions calculated by the adjacent iterations are employed as the predefined convergence tolerance $\omega=\varpi=v_{1}=v_{2}=10^{-5}$ of the proposed algorithm for the fair comparison. As shown in Fig. 4 and Fig. 5, the proposed algorithm with PS protocol can achieve near-optimal energy efficiency about 21.4573bits/J. For TS protocol, the energy efficiency of the proposed algorithm is about 23.94bits/J. Comparing with the exhaustive search method, we can find that the proposed algorithm can achieve a very small output performance loss. On the other hand, we can also find that the proposed algorithm with PS and TS protocols converges within 7 iterations. This result reflects that the proposed algorithm has lower computational complexity than that of the exhaustive search method. 
For the proposed algorithm and the exhaustive search method, Fig. 6 illustrates the achievable energy efficiency versus the distance between AP to relay $d_{A S}$ with different settings of circuit power consumption $P_{c}$. From Fig. 6, there is about $0.003 \mathrm{bit} / \mathrm{J}$ energy efficiency performance loss of the proposed algorithm. Especially, the energy efficiency of the proposed algorithm can achieve 33.62bits $/ \mathrm{J}$ when $P_{c}=10 \mathrm{~mW}$ and $d_{A S}=1.5 \mathrm{~m}$. With the increase of $d_{A S}$ from $1.2 \mathrm{~m}$ to $1.8 \mathrm{~m}$, the achievable energy efficiency increases. Besides, it can also be noticed that less circuit power consumption of the networks leads to higher energy efficiency.

For the TS protocol, the effects of the circuit power consumption and the distance between AP to relay on energy efficiency are illustrated in Fig. 7. Similar to PS protocol, the proposed algorithm can achieve similar output performance with the exhaustive search method. However, the proposed algorithm only requires a relatively lower complexity. The energy efficiency is increased with the improvement of $d_{A S}$ and the reduction of $P_{c}$. Specifically, the proposed algorithm can achieve 22.3bits $/ \mathrm{J}$ when $P_{c}=40 \mathrm{~mW}$ and $d_{A S}=1.8 \mathrm{~m}$.

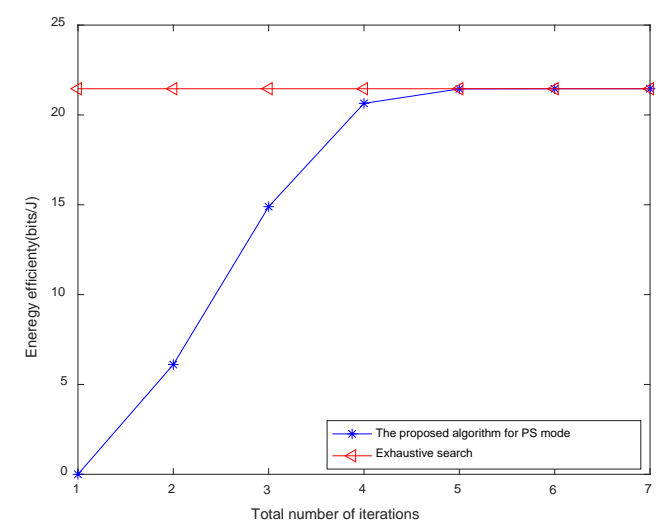

Fig. 4. Energy efficiency versus iteration numbers of PS protocol.

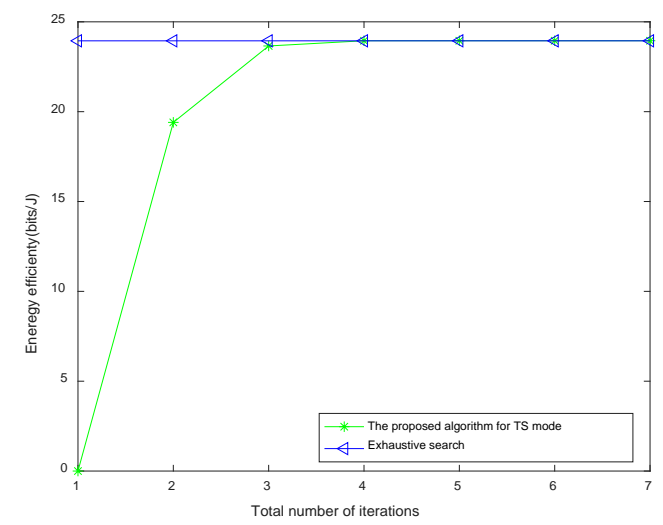

Fig. 5. Energy efficiency versus iteration numbers of TS protocol. 


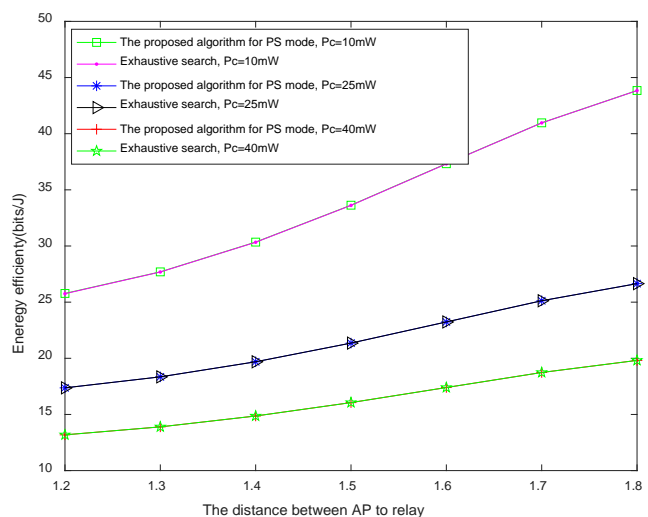

Fig. 6. Energy efficiency versus the distance between AP to relay of PS protocol.

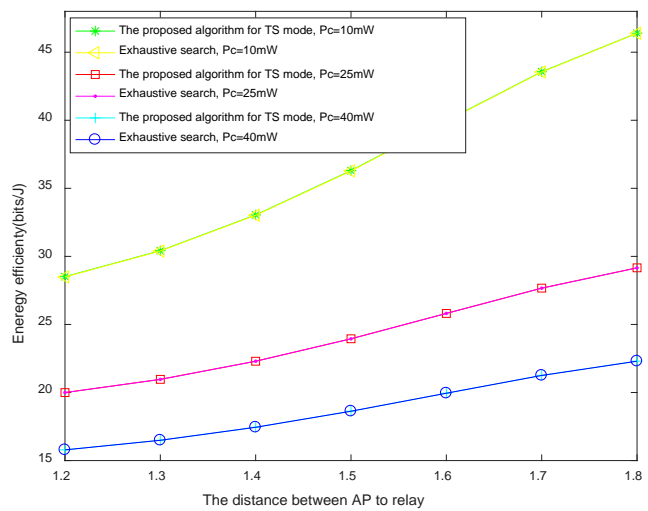

Fig. 7. Energy efficiency versus the distance between AP to relay of TS protocol.

In Fig. 8 and Fig. 9, we plot the energy efficiency under various resource division ratio with different transmission powers of AP $20 \mathrm{dBm}, 23 \mathrm{dBm}$ and $27 \mathrm{dBm}$, respectively. It can be seen that these six curves have the maximum energy efficiency with different resource division ratio. The energy efficiency first increases and then decreases as the resource division ratio increases. Moreover, energy efficiency can achieve 21.983bits $/ \mathrm{J}$ when $P_{A}=27 \mathrm{dBm}$ and $\rho=0.7$. Besides, energy efficiency can achieve 23.45bits $/ \mathrm{J}$ when $P_{A}=27 \mathrm{dBm}$ and $\alpha=0.124$.

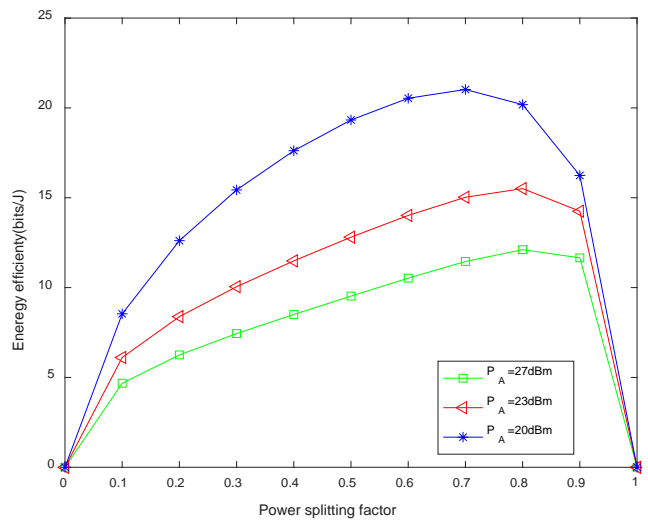

Fig. 8. Energy efficiency versus different PS factor when $P_{A}=27 \mathrm{dBm}, P_{A}=23 \mathrm{dBm}$ and $P_{A}=20 \mathrm{dBm}$. 


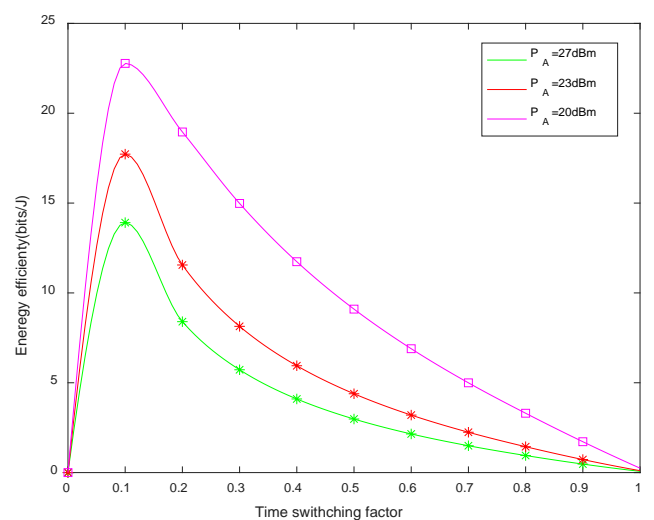

Fig. 9. Energy efficiency versus different TS factor when $P_{A}=27 \mathrm{dBm}, P_{A}=23 \mathrm{dBm}$ and $P_{A}=20 \mathrm{dBm}$.

With the minimum rate requirement varying from $R_{t h}=0 \mathrm{bps} / \mathrm{Hz}$ to $R_{t h}=3 \mathrm{bps} / \mathrm{Hz}$, Fig. 10 shows the effect of the path-loss exponent when $\lambda=2.5, \lambda=3$ and $\lambda=4$. The circuit power consumption and the distance between AP to relay are fixed at $P_{c}=25 \mathrm{~mW}$ and $d_{A S}=1.5 \mathrm{~m}$. As expected, the proposed PS scheme is declining about 20.79bits/J with the increasing path-loss exponent between $\lambda=2.5$ to $\lambda=4$. With the increasing of $R_{t h}$, achievable energy efficiency is declining. The reason is that a larger $R_{t h}$ may lead to smaller feasible domain of the optimization problem. When $R_{t h}$ becomes large enough, the information rate of the proposed algorithm is even less than the minimum rate requirement, which is another reason for excessively low energy efficiency.

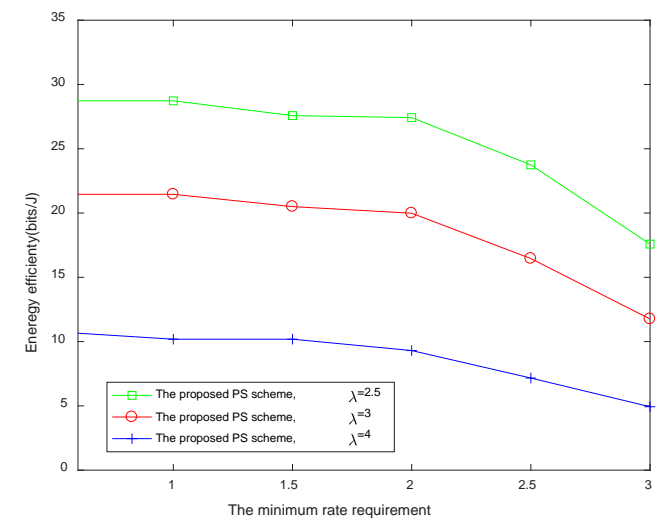

Fig. 10. Energy efficiency versus the minimum rate requirement of PS protocol.

For the TS protocol, the effects of $R_{t h}$ and $\lambda$ on energy efficiency are shown in Fig. 11. We can see that the energy efficiency of the proposed schemes decline if we expect to achieve a larger value of the minimum rate requirement. The reason for this phenomenon is similar to Fig. 10. Additionally, the output performance is reduced by the bigger path-loss exponent. That is because the bigger path-loss exponent indicates the stronger the channel fading. The energy efficiency can only achieve $3.0961 \mathrm{bits} / \mathrm{J}$ when $\lambda=4$ and $R_{t h}=3 \mathrm{bps} / \mathrm{Hz}$. 


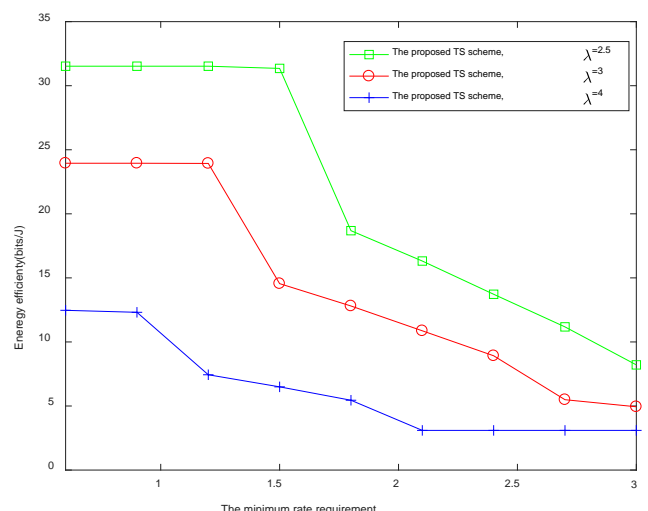

Fig. 11. Energy efficiency versus the minimum rate requirement of TS protocol.

In Fig. 12 and Fig. 13, we compare the proposed schemes with other conventional schemes and rate maximization scheme in [27] when the energy conversion efficiency varies from 0.1 to 0.9. Therefore, the first compared schemes are the optimal resource division ratio schemes with the fixed transmission power of AP $P_{A}=0.8$ and $P_{A}=0.5$. The second compared schemes are the optimal transmission with the fixed resource division ratio $\rho=0.3, \rho=0.5$, $\alpha=0.3$ and $\alpha=0.5$. Moreover, the third compared schemes are the rate maximization schemes with the optimal resource division ratio and the optimal transmission power [30].

As shown in Fig. 12 and Fig. 13, the proposed schemes with PS and TS protocols have the highest energy efficiency compared with the other three schemes. In particular, the energy efficiency of the proposed PS scheme can achieve $55.68 \mathrm{bits} / \mathrm{J}$ when $\xi=0.9$. In Fig. 13, the energy efficiency of the proposed TS scheme can achieve 48.32bits/J when $\xi=0.5$. Additionally, the optimization of resource division ratio and transmission power has an impact on energy efficiency. Moreover, the six energy efficiency curves are monotonously increasing as the energy conversion factor becomes larger. The reason is that the relay node and SN can harvest more energy for transmission.

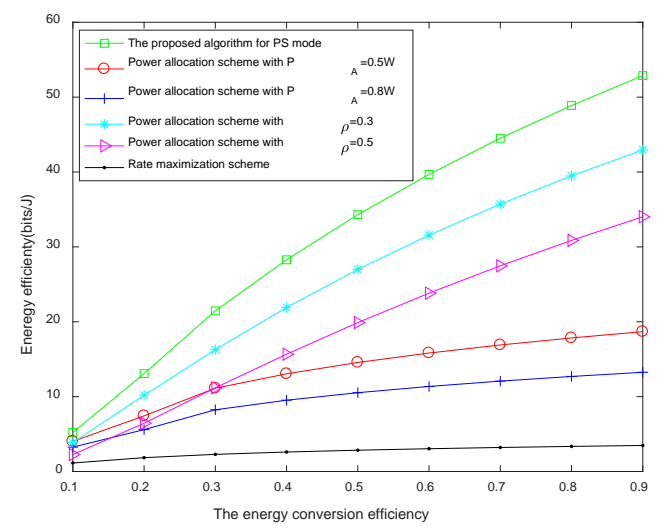

Fig. 12. Energy efficiency of the proposed PS scheme versus other schemes. 


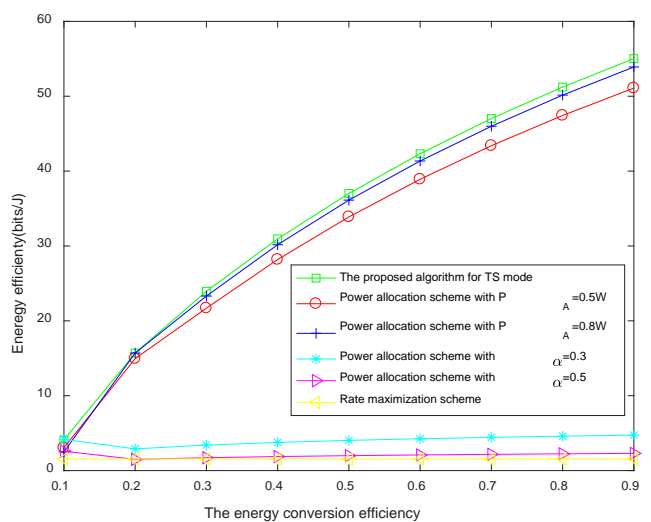

Fig. 13. Energy efficiency of the proposed TS scheme versus other schemes.

\section{Conclusion}

For bidirectional EH cooperative sensor networks, we investigate the energy efficiency maximization optimizations under TS and PS protocol, which can achieve uplink information transmission from the SN to AP and the downlink energy transmission from the AP to SN. Especially for the PS protocol, the impact of imperfect SIC on the system is studied. Considering the minimum $\mathrm{EH}$ activation constraint and minimum rate requirement, the non-convex energy efficiency optimizations are formulated with respect to the resource division ratio and the transmission power of AP. To cope with this difficulty, we propose the FAS algorithm based on fractional programming and alternative convex programming. Simulation results are conducted to verify the better output performance and convergence of the proposed schemes. In future work, multiple AP and SN pairs will be studied. Moreover, we will investigate the security problem of the considered network and merge physical layer security technology to prevent the eavesdropping of confidential information.

\section{Appendix}

\section{Proof of Proposition 1}

$P 1 a$ (32) can be re-expressed as

$$
\begin{aligned}
& \max _{\left\{P_{A}, \rho\right\}} \frac{1}{2} \log _{2}\left(1+\frac{A \rho^{2}(1-\rho) P_{A}}{B P_{A} \rho(1-\rho)+C \rho(1-\rho)+D(1-\rho)+E \rho}\right)-P_{\text {total }}^{P S}\left(P_{A}, \rho\right) \\
& \text { s.t. } \frac{1}{2} \log _{2}\left(1+\frac{A \rho^{2}(1-\rho) P_{A}}{B P_{A} \rho(1-\rho)+C \rho(1-\rho)+D(1-\rho)+E \rho}\right)>R_{t h}, \\
& \quad C 2-C 4
\end{aligned}
$$

where $A=\xi^{3}|h|^{6}|g|^{4}, B=\xi \zeta|h|^{6}, C=\sigma_{A}^{2} \xi^{2}|h|^{2}|g|^{4}, D=\sigma_{A}^{2} \zeta|h|^{2}$ and $E=\xi|h|^{4} \sigma_{R}^{2}$. With the fixed $\rho$, the second-order derivation of $\gamma_{P S}\left(P_{A}, \rho\right)$ with respect to $P_{A}$ can be calculated as 


$$
\frac{\partial^{2} R_{P S}\left(P_{A}, \rho\right)}{\partial P_{A}^{2}}=-\frac{2 A B C D E \rho^{5}(1-\rho)^{4}}{\left(B P_{A} \rho(1-\rho)+C \rho(1-\rho)+D(1-\rho)+F \rho\right)^{3}} .
$$

We can see that $\partial^{2} R_{P S}\left(P_{A}, \rho\right) / \partial P_{A}{ }^{2}$ less zero, which means that $R_{P S}\left(P_{A}, \rho\right)$ with respect to $P_{A}$ is a convex function. With the given $P_{A}$, the second-order derivation of $\gamma_{P S}\left(P_{A}, \rho\right)$ with respect to $\rho$ can be calculated as

$$
\begin{aligned}
& \frac{\partial^{2} \gamma_{P S}\left(P_{A}, \rho\right)}{\partial \rho^{2}} \\
& =-\frac{2 A \rho P_{A}\left(D^{2}\left(\rho^{3}-3 \rho^{2}+3 \rho-1\right)+E^{2} \rho^{3}+E \rho^{3}\left(B P_{A}+C\right)+D E \rho^{2}(3-2 \rho)\right)}{\left(D(1-\rho)+B P_{A} \rho(1-\rho)+C \rho(1-\rho)+E \rho\right)^{3}}
\end{aligned}
$$

In (38), we can prove that the terms $\rho^{3}-3 \rho^{2}+3 \rho-1$ and $3-2 \rho$ are positive within $\rho \in(0,1)$. Thus, $\gamma_{P S}\left(P_{A}, \rho\right)$ and $R_{P S}\left(P_{A}, \rho\right)$ with respect to $P_{A}$ and $\rho$ are two convex functions respectively. Besides, other constraints in (36) are also convex. Thus, the decomposed two sub-problems are convex.

\section{Proof of Proposition 2}

In $(k+1)$ th iterations, the local solution of $P_{A}(k+1)$ can be obtained with the given $\rho(k)$ by fast gradient method [41], while $P_{A}(k)$ is only the feasible solution. Hence, we have

$$
R_{P S}\left(P_{A}(k+1), \rho(k)\right)-\varphi P_{\text {total }}^{P S}\left(P_{A}(k+1), \rho(k)\right) \leq R_{P S}\left(P_{A}(k), \rho(k)\right)-\varphi P_{\text {total }}^{P S}\left(P_{A}(k), \rho(k)\right) .
$$

Similarly, $\rho(k+1)$ is the local optimal solution with the obtained $P_{A}(k+1)$, while $\rho(k)$ is only a feasible solution. We can conclude that

$$
\begin{aligned}
& R_{P S}\left(P_{A}(k+1), \rho(k+1)\right)-\varphi P_{\text {total }}^{P S}\left(P_{A}(k+1), \rho(k+1)\right) \\
& \leq R_{P S}\left(P_{A}(k+1), \rho(k)\right)-\varphi P_{\text {total }}^{P S}\left(P_{A}(k+1), \rho(k)\right)
\end{aligned} .
$$

Adding the equation (39) and equation (40), we can obtain that

$$
\begin{aligned}
& R_{P S}\left(P_{A}(k+1), \rho(k+1)\right)-\varphi P_{\text {total }}^{P S}\left(P_{A}(k+1), \rho(k+1)\right) \\
& \leq R_{P S}\left(P_{A}(k), \rho(k)\right)-\varphi P_{\text {total }}^{P S}\left(P_{A}(k), \rho(k)\right)
\end{aligned} .
$$

After obtaining (41), it means that the sequence $R_{P S}\left(P_{A}(k), \rho(k)\right)-\varphi P_{\text {total }}^{P S}\left(P_{A}(k), \rho(k)\right)$ is monotonically decreasing and convergent. 


\section{Proof of Proposition 3}

For simplicity, $P 2 a$ (35) can be re-expressed as

$$
\begin{aligned}
& \max _{\left\{P_{A}, \alpha\right\}} \frac{1-\alpha}{2} \log _{2}\left(1+\frac{A_{1} P_{A} \alpha}{B_{1}(1-\alpha)}\right)-P_{\text {total }}^{T S}\left(P_{A}, \alpha\right) \\
& \text { s.t. } \quad \frac{1-\alpha}{2} \log _{2}\left(1+\frac{A_{1} P_{A} \alpha}{B_{1}(1-\alpha)}\right)>R_{\text {th }} \\
& \\
& \quad C 2-C 4
\end{aligned}
$$

where $A_{1}=2 \xi|h|^{4}|g|^{4}$ and $B_{1}=|h|^{2} \sigma_{R}^{2}+\xi|g|^{4} \sigma_{A}^{2}$. With the fixed $\alpha$ or $P_{A}$, the second-order derivation of $R_{T S}\left(P_{A}, \alpha\right)$ with respect to $P_{A}$ and $\alpha$ can be calculated as

$$
\begin{gathered}
\frac{\partial^{2} R_{T S}\left(P_{A}, \alpha\right)}{\partial P_{A}^{2}}=-\frac{1-\alpha}{2} \frac{A_{1}^{2} \alpha^{2}}{\ln 2\left(A_{1} P_{A} \alpha+B_{1}(1-\alpha)\right)^{2}} . \\
\frac{\partial^{2} R_{T S}\left(P_{A}, \alpha\right)}{\partial \alpha^{2}}=-\frac{A^{2}}{2 \ln 2(1-\alpha)(A \alpha+-B(1-\alpha))^{2}} .
\end{gathered}
$$

It can be seen that both (43) and (44) are negative. Therefore, $R_{T S}\left(P_{A}, \alpha\right)$ is convex for $\alpha$ with fixed $P_{A}$, and $R_{T S}\left(P_{A}, \alpha\right)$ is also convex for $P_{A}$ with fixed $\alpha$. Hence, the decomposed two sub-problems are two convex problems since the constraints in (42) are also convex.

\section{References}

[1] F. Hu, X. Liu, M. Shao, D. Sui and L. Wang, "Wireless energy and information transfer in WBAN: An overview," IEEE Network, vol. 31, pp. 90-96, May, 2017. Article (CrossRef Link)

[2] D. Du, H. Fengye, F. Wang, Z. Wang, Y. Du and L. Wang, "A game theoretic approach for inter-network interference mitigation in wireless body area networks," China Communications, vol. 12, no. 9, pp. 150-161, September, 2015. Article (CrossRef Link)

[3] B. Medepally and N. Mehta, "Voluntary energy harvesting relays and selection in cooperative wireless networks," IEEE Transactions on Wireless Communications, vol. 9, no. 11, pp. 3543-3553, November, 2010. Article (CrossRef Link)

[4] L. R. Varshney, "Transporting information and energy simultaneously," in Proc. of IEEE Int. Symp. Inf. Theory (ISIT), pp. 1612-1616, July 6-11, 2008. Article (CrossRef Link)

[5] K.-H. Liu and P. Lin, "Toward self-sustainable cooperative relays: State of the art and the future," IEEE Communications Magazine, vol. 53, no. 6, pp. 56-62, June, 2015. Article (CrossRef Link)

[6] L. Liu, R. Zhang and K.-C. Chua, "Wireless information and power transfer: A dynamic power splitting approach," IEEE Transactions on Communications, vol. 61, no. 9, pp. 3990-4001, September, 2013. Article (CrossRef Link)

[7] A. A. Nasir, X. Zhou, S. Durrani and R. A. Kennedy, "Relaying protocols for wireless energy harvesting and information processing," IEEE Transactions on Wireless Communications, vol. 12, no. 7, pp. 3622-3636, July, 2013. Article (CrossRef Link) 
[8] A. A. Nasir, X. Zhou, S. Durrani and R. A. Kennedy, "Throughput and ergodic capacity of wireless energy harvesting based DF relaying network," in Proc. of IEEE Int. Conf. Commun. (ICC), pp. 4066-4071, June 10-14, 2014. Article (CrossRef Link)

[9] I. Ahmed, I. Ahmed and J. Hossain, "Optimal stochastic power allocation and relay selection for energy harvesting systems,” IEEE Wireless Communications Letters, vol. 6, no. 4, pp. 546-549, August, 2017. Article (CrossRef Link)

[10] P. Ramezani and A. Jamalipour, "Toward the evolution of wireless powered communication networks for the future internet of things," IEEE Network, vol. 31, no. 6, pp. 62-69, November/December, 2017. Article (CrossRef Link)

[11] X. Gao, P. Wang, D. Niyato, K. Yang and J. An, "Auction-based time scheduling for backscatter-aided RF-powered cognitive radio networks," IEEE Transactions on Wireless Communications, vol. 18, no. 3, pp. 1684-1697, March, 2019. Article (CrossRef Link)

[12] X. Gao, D. Niyato, P. Wang, K. Yang and J. An, “Contract design for time resource assignment and pricing in backscatter-assisted RF-powered networks," IEEE Wireless Communications Letters, vol. 9, no. 1, pp. 42-46, January, 2020. Article (CrossRef Link)

[13] X. Gao, P. Wang, D. Niyato, K. Yang and J. An, “An Auction-Based Time Scheduling Mechanism for Backscatter-Aided RF-Powered Cognitive Radio Networks,” in Proc. of IEEE Int. Conf. Internet Things, pp. 301-307, July 30-August 3, 2018. Article (CrossRef Link)

[14] B. Li and Z. Fei, "Probabilistic-constrained robust secure transmission for energy harvesting over MISO channels," Science China(Information Sciences), vol. 61, no.2, pp. 65-76, 2018. Article (CrossRef Link)

[15] B. Li, Z. Fei, Z. Chu and Y. Zhang, "Secure Transmission for Heterogeneous Cellular Networks With Wireless Information and Power Transfer,” IEEE Systems Journal, vol. 12, no. 4, pp. 3755-3766, December, 2018. Article (CrossRef Link)

[16] B. Li, Z. Fei and H. Chen, "Robust Artificial Noise-Aided Secure Beamforming in Wireless-Powered Non-Regenerative Relay Networks,” IEEE Access, vol. 4, pp. 7921-7929, November, 2016. Article (CrossRef Link)

[17] L. Shi, Y. Ye, X. Chu, Y. Zhang and H. Zhang, "Optimal Combining and Performance Analysis for Two-Way EH Relay Systems With TDBC Protocol,” IEEE Wireless Communications Letters, vol. 8, no. 3, pp. 713-716, June, 2019. Article (CrossRef Link)

[18] N. T. P. Van, S. F. Hasan, X. Gui, S. Mukhopadhyay and H. Tran, "Three-step two-way decode and forward relay with energy harvesting," IEEE Communications Letters, vol. 21, no. 4, pp. 857-860, April, 2017. Article (CrossRef Link)

[19] L. Shi, Y. Ye, R. Q. Hu and H. Zhang, "Energy Efficiency Maximization for SWIPT Enabled Two-Way DF Relaying,” IEEE Signal Processing Letters, vol. 26, no. 5, pp. 755-759, May, 2019. Article (CrossRef Link)

[20] L. Shi, Y. Ye, R. Q. Hu and H. Zhang, "System outage performance for three-step two-way energy harvesting DF relaying," IEEE Transactions on Vehicular Technology, vol. 68, no. 4, pp. 3600-3612, April, 2019. Article (CrossRef Link)

[21] L. Shi, W. Chen, Y. Ye, H. Zhang and R. Hu, "Heterogeneous power-splitting based two-way DF relaying with non-linear energy harvesting," in Proc. of IEEE Global Commun. Conf., pp. 1-7, December 9-13, 2018. Article (CrossRef Link)

[22] J. Men, J. Ge, C. Zhang and J. Li, "Joint optimal power allocation and relay selection scheme in energy harvesting asymmetric two-way relaying system,” IET Communications, vol. 9, no. 11, pp. 1421-1426, July, 2015. Article (CrossRef Link)

[23] Y. Zhang, J. Ge, J. Men, F. Ouyang and C. Zhang, "Joint relay selection and power allocation in energy harvesting AF relay systems with ICSI,” IET Microwaves, Antennas \& Propagation, vol. 10, no. 15, pp. 1656-1661, December, 2016. Article (CrossRef Link)

[24] X. Song, S. Xu, Z. Xie and X. Han "Joint optimal power allocation and relay selection scheme in energy harvesting two-way relaying network,” Future Internet, vol. 11, p.47, February, 2019. Article (CrossRef Link) 
[25] D. Li, C. Shen and Z. Qiu, "Sum rate maximization and energy harvesting for two-way AF relay systems with imperfect CSI," in Proc. of IEEE Int. Conf. Acoust. Speech Signal Process. (ICASSP), pp. 4958-4962, May 26-31, 2013. Article (CrossRef Link)

[26] D. Li, C. Shen and Z. Qiu, “Two-way relay beamforming for sum-rate maximization and energy harvesting,” in Proc. of IEEE Int. Conf. Commun. (ICC), pp. 3120-3155, June 9-13, 2013. Article (CrossRef Link)

[27] R. Chen and H. Zhang, "Decode-and-forward relay based bidirectional wireless information and power transfer,” China Communications, vol. 14, no. 8, pp. 176-183, August, 2017. Article (CrossRef Link)

[28] R. Chen, H. Zhang and Y. Zhou, "Bidirectional wireless information and power transfer for decode-and-forward relay systems,” IET Signal Processing, vol. 11, no. 8, pp. 1015-1020, October, 2017. Article (CrossRef Link)

[29] R. Chen, Y. Sun, Y. Chen, X. Zhang, S. Li and Z. Sun, "Energy Efficiency Analysis of Bidirectional Wireless Information and Power Transfer for Cooperative Sensor Networks,” IEEE Access, vol. 7, pp. 4905-4912, December, 2019. Article (CrossRef Link)

[30] Y. Zeng, H. Chen and R. Zhang, "Bidirectional wireless information and power transfer with a helping relay,” IEEE Communications Letters, vol. 20, no. 5, pp. 862-865, May, 2016. Article (CrossRef Link)

[31] L. Xiang, X. Ge, C. Wang, F. Y. Li and F. Reichert, "Energy Efficiency Evaluation of Cellular Networks Based on Spatial Distributions of Traffic Load and Power Consumption," IEEE Transactions on Wireless Communications, vol. 12, no. 3, pp. 961-973, March, 2013. Article (CrossRef Link)

[32] X. Ge, B. Yang, J. Ye, G. Mao, C. Wang and T. Han, "Spatial Spectrum and Energy Efficiency of Random Cellular Networks,” IEEE Transactions on Communications, vol. 63, no. 3, pp. 1019-1030, March, 2015. Article (CrossRef Link)

[33] X. Ge, Y. Sun, H. Gharavi and J. Thompson, "Joint Optimization of Computation and Communication Power in Multi-User Massive MIMO Systems,” IEEE Transactions on Wireless Communications, vol. 17, no. 6, pp. 4051-4063, June, 2018. Article (CrossRef Link)

[34] D. Wang, B. Bai, W. Zhao and Z. Han, “A survey of optimization approaches for wireless physical layer security,” IEEE Communications Surveys \& Tutorials, vol. 21, no. 2, pp. 1878-1911, November, 2019. Article (CrossRef Link)

[35] W. Cheng, X. Zhang and H. Zhang, "Statistical-QoS driven energy-efficiency optimization over green 5G mobile wireless networks,” IEEE Journal on Selected Areas in Communications, vol. 34, no. 12, pp. 3092-3107, December, 2016. Article (CrossRef Link)

[36] D. Wang, B. Bai, W. Chen and Z. Han, "Secure green communication for amplify-and-forward relaying with eavesdroppers," in Proc. of IEEE Int. Conf. Commun. (ICC), pp. 4468-4473, June 8-12, 2015. Article (CrossRef Link)

[37] D. Wang, B. Bai, W. Chen and Z. Han, "Energy efficient secure communication over decode-and-forward relay channels," IEEE Transactions on Communications, vol. 63, no. 3, pp. 892-905, March, 2015. Article (CrossRef Link)

[38] D. Wang, B. Bai, W. Chen and Z. Han, “Achieving high energy efficiency and physical-layer security in AF relaying," IEEE Transactions on Wireless Communications, vol. 15, no. 1, pp. 740-752, January. 2016. Article (CrossRef Link)

[39] W. Dinkelbach, “On nonlinear fractional programming,” Management Science, vol. 13, no. 7, pp. 435-607, March. 1967. Article (CrossRef Link)

[40] S. Schaible, "Fractional programming. II on Dinkelbach's algorithm", Management Science, vol. 22, no. 8, pp. 829-925, April. 1976. Article (CrossRef Link)

[41] S. Richter, C. N. Jones and M. Morari, “Computational complexity certification for real-time MPC with input constraints based on the fast gradient method," IEEE Transactions on Automatic Control, vol. 57, no. 6, pp. 1391-1403, June, 2012. Article (CrossRef Link) 


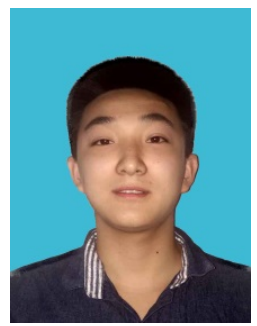

Siyang Xu received the B.E. degree in Electronics and Information Engineering from the University of Science and Technology Liaoning, China, 2016 and the M.S. degree in Computer Science and Engineering department from Northeastern University (NEU), Shenyang, China in 2018. He is currently pursuing the Ph.D. degree in Communication and Information System with Northeastern University (NEU), Shenyang, China. His current research interests include energy harvesting, physical layer security and relay cooperative communication.

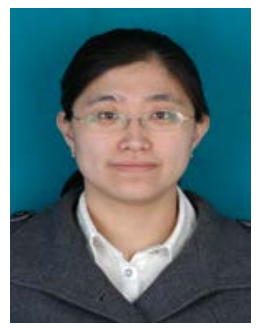

Xin Song was born in Jilin, China, in 1978. She received her Ph.D. degree in Communication and Information System in Northeastern University in China in 2008. She is now a teacher working in Northeastern University at Qinhuangdao, China. Her research interests are in the area of robust adaptive beam-forming and wireless communication.

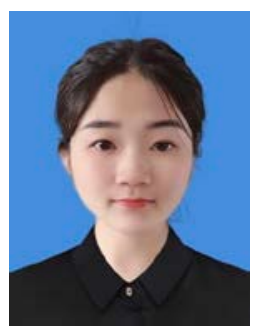

Lin Xia received the B.E. degree in Electronics and Information Engineering from the University of Science and Technology Liaoning, China, 2018. She is currently pursuing the M. S. degree in Electronic and communications engineering with Northeastern University (NEU), Shenyang, China. Her current research interests include full-duplex device-to-device communications, physical layer security and wireless resource allocation.

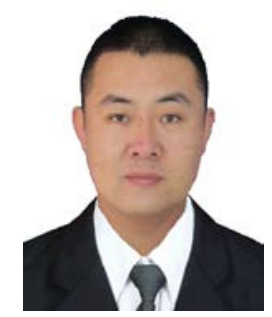

Zhigang Xie received the B.E. degree in communications engineering from Northeastern University at Qinhuangdao (NEUQ), Qinhuangdao, China in 2012 and the M.E. degree in Software Engineering from Northeastern University (NEU), Shenyang, China in 2016. He is currently working towards his Ph.D. degree in Communication and Information System at Northeastern University, Shenyang, China. His research interests include 5G network and mobile edge computing. 